\title{
A Raum With a View: \\ Hermann Weyl and the Problem of Space
}

\author{
Neil Dewar and Joshua Eisenthal
}

April 14, 2019

\section{Introduction}

One of the central philosophical debates prompted by general relativity concerns the status of the metric field. A number of philosophers have argued that the metric field should no longer be regarded as part of the background arena in which physical fields evolve; it should be regarded as a physical field itself. Earman and Norton write, for example, that the metric tensor in general relativity 'incorporates the gravitational field and thus, like other physical fields, carries energy and momentum'.1 Indeed, they baldly claim that according to general relativity 'geometric structures, such as the metric tensor, are clearly physical fields in spacetime'. ${ }^{2}$ On such a view, spacetime itselfconsidered independently of matter-has no metrical properties, and the mathematical object that best represents spacetime is a bare topological manifold. As Rovelli puts the idea: 'the metric/gravitational field has acquired most, if not all, the attributes that have characterized matter (as opposed to spacetime) from Descartes to Feynman... it is perhaps more appropriate to reserve the expression spacetime for the differential manifold, and to use the expression matter for everything which is dynamical, carries energy and so on; namely all the fields including the gravitational field.' ${ }^{3}$

Others, however, have strongly resisted this view, arguing that the paradigm spatiotemporal properties are precisely the metrical properties. Thus Tim Maudlin has written:

1(Earman and Norton, 1987, p. 519)

${ }^{2}$ (Earman and Norton, 1987, p. 519)

3 (Rovelli, 1997, pp. 193-194) 
...qua differentiable manifold, abstracting from the metrical (and affine) structure, space-time has none of the paradigm spatio-temporal properties. The light-cone structure is not defined; past and future cannot be distinguished; distance relations do not exist. Spatio-temporal structure is metrical structure. ${ }^{4}$

In resisting the adoption of a bare-manifold account of spacetime Maudlin has been joined by Carl Hoefer, who has argued that the mere fact that the metric field appears to carry energy does not imply that it should be regarded as a physical field. ${ }^{5}$ More specifically, Hoefer argued that the complexities arising from the fact that gravitational stress-energy is represented by a pseudo-tensor cannot be merely brushed aside, and that there are good reasons to be skeptical of any quick inferences based on the existence of gravitational energy in this context. ${ }^{6}$

This debate-between bare-manifold and manifold-plus-metric accounts of spacetimetouches on the long-standing philosophical problem of how mathematics represents the world. In the context of geometry, the most immediate aspect of this problem is the "Problem of Space": the problem of determining which abstract geometrical structures are candidate physical geometries, i.e., candidate descriptions of physical space. It is only since the advent of non-Euclidean geometries that this problem has emerged, or could even be stated. For most of its history, of course, geometry was just Euclidean geometry, understood as the systematic description of spatial structure ('the most ancient branch of physics', as Einstein once put it). ${ }^{7}$ Hence it was only after the existence of non-Euclidean geometries was grudgingly accepted that it became possible to ask: which geometry actually describes space? By the end of the nineteenth century (as we summarize below) consensus formed around the following answer: the candidate physical geometries are the constant curvature geometries; the geometries in which congruence relations can represent the free mobility of rigid bodies.

However, this "classical" solution to the Problem of Space was unequivocally undermined by general relativity - the theory of spacetime that employs precisely the kind of variably curved geometry that the philosophers of the nineteenth century thought they had ruled out. In this paper, we explore the new solution to the Problem of Space advanced by Hermann Weyl, drawing especially on the account Weyl gave of this prob-

${ }^{4}$ (Maudlin, 1988, p. 87; emphasis added)

${ }^{5}$ Cf. Hoefer (1996)

${ }^{6}$ For further discussion of the controversies over gravitational energy, see Curiel (2017), Read (2018), and Dewar and Weatherall (2018).

${ }^{7}$ Einstein (1921) 
lem in a series of lectures delivered in Barcelona in 1922. ${ }^{8}$ Weyl had an exceptionally nuanced understanding of the novel conception of spacetime implicit in general relativity, and our concrete goal in what follows is to show that an important insight made available by Weyl's work is the unearthing of a "middle way" between bare-manifold and manifold-plus-metric accounts of spacetime.

\section{The Classical Solution}

The classical solution to the Problem of Space is, in effect, the fruits of the cumulative effort of those who engaged with the problem in the second half of the nineteenth century. The formulation of the Problem of Space in this context is often referred to as the Helmholtz (or Helmholtz/Lie) space problem, but as Helmholtz himself pointed out it had already been treated substantially by Riemann. Helmholtz's results were rigorously reworked and extended by Sophus Lie, who brought to bear the full power of his theory of continuous transformation groups. ${ }^{9}$ Poincare also grappled with the Problem of Space, and although his philosophical stance differed significantly from Helmholtz's, he clearly regarded the problem as more-or-less solved once Lie had put Helmholtz's arguments on a sufficiently rigorous footing. ${ }^{10}$

The essential idea that emerged in this period was that the geometrical notion of congruence represented the possibility of the free mobility of rigid bodies. Treating such free mobility as a basic fact (and recognising the role that this fact seemed to play in the practice of measurement quite generally), a limited class of geometries could be specified as candidate descriptions of physical space. In particular, it was argued that only in constant curvature geometries were there suitable congruence relations between geometric figures to represent the free mobility of rigid bodies.

The close connection between congruence relations of geometrical figures and constant curvature was proved most rigorously by Lie who considered the properties of the real, finite-dimensional transformation groups that correspond to classical congruence relations, but it is also easy to understand the basic point intuitively. Helmholtz discussed the example of the non-constant curvature of an egg-shaped surface, observing

\footnotetext{
${ }^{8}$ Weyl (1923a)

${ }^{9}$ For Riemann and Helmholtz, the problem at hand was one in which physics and mathematics necessarily intertwined. In contrast, Lie treated the problem as the purely mathematical one of rigorously characterizing distinct classes of geometrical structures. The articulation of the range of mathematical possibilities was developed also by Klein, Clifford and Killing: see Scholz (2016), p. 5 for a summary of this work.

${ }^{10}$ Poincaré remarked, 'I differ from [Helmholtz and Lie] in one point only, but probably the difference is in the mode of expression only and at bottom we are completely in accord' (Poincaré, 1898, p. 40).
} 
that a figure such as a triangle would have different internal angles if it were drawn near the pointed end of the egg, compared to if it were drawn near the base. ${ }^{11}$ Similarly, two circles with the same radius would not in general have the same circumference. Thus sliding any such figure up or down the surface would not be possible unless it were flexible enough to change its dimensions as it moved. In the general case: in any space of varying curvature (with zero degrees of symmetry), a truly rigid figure-one that could not alter its dimensions without breaking - would not be able to be moved at all.

In this way, Helmholtz, and Poincaré after him, argued that geometries of non-constant curvature are not feasible candidates for describing physical space at all. Their reasoning depended on the premise that constructing a physical geometry depends essentially on the use of rigid bodies. According to Helmholtz, this is a generalization of the requirement that material measuring instruments (rulers, compasses, and the like) must maintain their dimensions if they are to fulfill their function. ${ }^{12}$ Helmholtz argued that if there were no rigid bodies, and hence no way of comparing spatial magnitudes, it would not be possible to construct any kind of physical geometry. Hence variably curved geometries, which lack the relevant congruence structure, cannot provide a useful description of physical space (for even if we lived in such a space, so the thought goes, we would not be able to construct a geometrical description of it). Thus Helmholtz declared: 'all original spatial measurement depends on asserting congruence and therefore, the system of spatial measurement must presuppose the same conditions on which alone it is meaningful to assert congruence'. ${ }^{13}$ Poincaré, for his part, said of variably curved geometries that they could 'never be anything but purely analytic, and they would not be susceptible to demonstrations analogous to those of Euclid'. ${ }^{14}$

This, then, was the classical solution to the Problem of Space: a geometrical structure could describe spatial structure only insofar as it could represent the free mobility of rigid bodies. Combined with Lie's work, this postulate of free mobility implied a clear demarcation of candidate physical geometries from two directions. First, candidate physical geometries could only be constant curvature geometries. Second, the metric function must satisfy a generalized Pythagorean Theorem, i.e. the element of length must be given by the square root of a quadratic differential form.

However, the development of relativity-particularly general relativity-broke the back of this purported solution to the Problem of Space. One obvious change due to relativity was the new way in which space and time were welded together into

\footnotetext{
${ }^{11}$ Cf. Helmholtz (1971)

${ }^{12}$ Cf. (Helmholtz, 1971, p. 63)

${ }^{13}$ (Helmholtz, 2007, p. 49)

14(Poincaré, 1952, p. 103)
} 
spacetime, but the more immediately significant change was actually the threat to the notion of a rigid body. Already in special relativity it became clear that perfectly rigid bodies were simply incompatible with the theory. ${ }^{15}$ But it was only with the advent of general relativity that the classical solution to the Problem of Space was definitively undermined, for it is general relativity that employs precisely the variably curved geometrical structure that Helmholtz and Poincaré had exiled from the class of candidate physical geometries. As Scholz has put the matter, general relativity 'posed, of course, a much greater challenge to the characterization of the Problem of Space. Free mobility of finitely extended rigid figures became meaningless in the general case. ${ }^{\prime 16}$

\section{Weyl's Problem of Space}

In the wake of general relativity it is evident that the classical demarcation of candidate physical geometries is too restrictive-clearly, an adequate demarcation must include variably curved geometries too. This is the context in which Weyl sought to justify a new and broader conception of physical geometry. On Weyl's view, the possibility of describing physical space in geometrical terms depends only on the possibility of infinitesimal comparisons of lengths and angles. This still allows for the construction of practically rigid bodies, so that, as long as circumstances are not too hostile, we can still survey the meso-scale structure of space in the way that Helmholtz and others envisaged. But Weyl's solution also leaves room for the possibility of describing the geometry of a region of space encompassing such strongly varying gravitational fields that surveying it with rigid measuring instruments would be impossible.

An important upshot of Weyl's approach to the Problem of Space is a distinction between the nature of the metric field, on the one hand, and the orientation of the metric field from point to point, on the other. The former is what determines the relative lengths of vectors at an arbitrary point, whilst the latter is what determines the relative lengths and angles of finitely separated vectors. Weyl uses this distinction to attribute the local metric properties to space itself, whilst attributing the non-local metric properties to the contingent distribution of matter and energy. In brief, rather than starting with the classical postulate of free mobility, Weyl starts with what he calls the 'foundational fact of infinitesimal geometry ${ }^{\prime 17}$ - the idea that the notion of congruent transport (the transformation that preserves length) uniquely determines a notion of affine transport

\footnotetext{
${ }^{15}$ For a more detailed discussion of the impact of special relativity on the notion of rigid bodies, see (Scholz, 2016, pp. 6-8).

${ }^{16}($ Scholz, 2016, p. 9)

${ }^{17}$ (Weyl, 1923b, p. 124), quoted at more length below.
} 
(the transformation that preserves parallelism). Weyl then proves that this foundational fact provides the basis for a new demarcation of physical geometries. According to Weyl, the candidate physical geometries are just the geometries with a PythagoreanRiemannian "nature"; the geometries whose metrics have an infinitesimal Pythagorean form.

It seems that Weyl first became interested in the Problem of Space when he was called upon to edit Riemann's 1868 Habilitationsvortrag for republication, for which he also provided a commentary. ${ }^{18}$ In the Habilitationsvortrag, Riemann gives the length of a line element as the square root of a quadratic form in the differentials, but remarks that this is merely the simplest case of a wider range of possibilities:

The next simplest case would probably comprise those manifolds in which the line element may be expressed by the fourth root of a differential expression of the fourth degree. To be sure, the investigation of this more general kind would not require any essentially different principles, but it would be rather time-consuming and would shed relatively little new light on the theory of space (especially as the results are not geometrically expressible); I therefore restrict myself to those manifolds where the line element is expressed by the quadratic root of a differential expression of the second degree. ${ }^{19}$

Weyl expresses Riemann's observation as follows: if the interval at point $P$ is expressed as a function of the differentials, $d s=f_{P}\left(d x_{1}, \ldots, d x_{n}\right)$, then ' $f_{P}$ will be required to be a homogeneous function of the first degree, in the sense that upon multiplication of the arguments $d x_{i}$ by a common real proportionality factor $\rho$, the function $f_{P}$ is multiplied by $|\rho|{ }^{\prime 20}$ An example of such a function is the familiar Pythagorean function:

$$
\sqrt{\left(d x_{1}\right)^{2}+\left(d x_{2}\right)^{2}+\cdots+\left(d x_{n}\right)^{2}}
$$

Up to a choice of coordinates, any function given as a square root of some positivedefinite quadratic form can be expressed in the form (1) (i.e. if $f_{P}^{2}$, at each point $P$, is a positive-definite quadratic form, then all the various $f_{P}$ can be obtained from the function (1) by linear transformations of the variables). However, (1) is not the

\footnotetext{
${ }^{18}$ Riemann and Weyl (1919)

${ }^{19}$ (Riemann and Weyl, 1919, p. 9); (Riemann and Jost, 2013, p. 35)

${ }^{20}$ (Riemann and Weyl, 1919, p. 26). In modern terminology, a homogeneous function is one for which $f(\lambda v)=\lambda f(v)$, whereas a function with the property that Weyl describes (being such that $f(\lambda v)=|\lambda| v)$ is described as absolutely homogeneous. In what follows, we will use 'homogeneity' in the same manner as Weyl (i.e. as a term for what is now called absolute homogeneity).
} 
only homogeneous function of the first degree, and so the question arises: why use this function to define intervals, rather than any others? Riemann himself offered no satisfactory justification for why the expression for the square of the line element should be a quadratic form, and hence it was a signature achievement of the classical solution to the Problem of Space to show that, if every physical geometry must represent the free mobility of rigid bodies, every physical geometry must have a Pythagorean metric. But when general relativity undermined the classical solution to the Problem of Space, this justification for the Pythagorean form of the metric vanished with it.

Thus, if we follow Weyl and say that the nature of a (Weylian) metric is given by specifying the expression for the line element, then the problem at hand is to justify the Pythagorean nature in particular. Weyl's solution to this problem begins from his generalisation of Riemannian geometry. ${ }^{21}$ In a Riemannian space $(M, g)$, where $M$ is a manifold and $g$ is a metric, whether two tangent vectors $v \in T_{p} M$ and $w \in T_{q} M$ (for finitely separated points $p$ and $q$ of $M$ ) are parallel is not—in general—determined absolutely, but only relative to the choice of a path connecting $p$ and $q$. This becomes most apparent if we introduce the concept of an affine connection following Levi-Civita. ${ }^{22}$. An affine connection establishes the parallelism-facts amongst the vectors in infinitesimally separated tangent spaces; it is only in the special case of a flat affine connection, however, that these can be extended to establish absolute (i.e. path-independent) parallelism-facts between vectors in finitely separated tangent spaces. ${ }^{23}$

For Weyl, Levi-Civita's work presented a profound insight into the structure of Riemannian geometry. But he soon recognised that it pointed to a way in which Riemannian geometry was not as local as it could be. Although Riemannian geometry does away with an absolute notion of distant parallelism, it retains an absolute notion of distant length-comparison: there is a always a definite answer to the question whether vectors $v \in T_{p} M$ and $w \in T_{q} M$ are the same length or not, even for finitely separated $p$ and $q$. More generally, for any two tangent vectors, there is some fact of the matter about their lengths, and hence about their length-ratio.

In aiming for a truly local geometry, then, our first move should be to do away with the structure of Riemannian geometry which permits such comparisons. Consider a pair of conformally equivalent metrics on $M$ : that is, metrics $g_{a b}$ and $g_{a b}^{\prime}$ such that for some

\footnotetext{
${ }^{21}$ Weyl (1918a), Weyl (1918b).

22 (Levi-Civita, 1917)

${ }^{23}$ Both Levi-Civita and Weyl took the term 'affine connection' to refer exclusively to symmetric (i.e. torsionfree) affine connections. We will have cause, however, to consider nonsymmetric affine connections in section 4 , so we will use the term 'affine connection' to refer to the broader class of such connections (whether symmetric or nonsymmetric).
} 
positive, nowhere-vanishing scalar field $\lambda: M \rightarrow \mathbb{R}$,

$$
g_{a b}^{\prime}=\lambda g_{a b} .
$$

A conformal structure on $M$ is an equivalence class of conformally equivalent metrics; a conformal manifold is a manifold equipped with a conformal structure. ${ }^{24}$ As Weyl remarks, in a conformal geometry the inner product of two vectors (in the same tangent space) is 'not absolute, but rather determined only up to an arbitrary non-zero proportionality factor'.25

Conformal manifolds do not permit distant length-comparisons: we can only determine the length-ratio between two vectors if they are drawn from the same tangent space. But in the passage to conformal manifolds, we have removed more structure than we wished to. For the analogy to parallelism (in Riemannian geometry) to hold, we don't want determinations of length-ratio between distant vectors to be impossible: we just want them to be relative to a choice of path. Equivalently, we want there to be (absolute) facts about the length-ratios of infinitesimally separated vectors, just not about the length-ratios of finitely separated vectors.

We must therefore restore the kind of structure that will let us make such comparisons, i.e., something analogous to an affine connection but for lengths rather than directions: 'a concept of transfer of the length unit from a point $P$ to its immediate neighbours'. ${ }^{26}$ Let us refer to such a standard of length transfer as a length connection. ${ }^{27}$ By transferring an (arbitrarily chosen) length unit from one point to another, we can compare the lengths of vectors in the tangent spaces at the two points, and the length connection operates in such a way that this length-ratio is independent of the choice of unit. Thus, in Weylian geometry, length-comparisons of distant vectors is once again possible but-in general—only relative to a path. As is the case for affine connections, things may work out such that the value of the integral is path-independent; in this case, we say that the length connection is flat, and the geometry is that of a Riemannian manifold up to an arbitrary global choice of length-scale. We will refer to a conformal structure together

\footnotetext{
${ }^{24}$ There is a natural sense, using the language of category theory, in which a conformal manifold is less structured than a Riemannian manifold. Let the category of Riemannian manifolds have isometries as arrows (and Riemannian manifolds as objects), and let the category of conformal manifolds have conformal maps as arrows (and either conformal manifolds or Riemannian manifolds as objects). Then there is a functor from the category of Riemannian manifolds to the category of conformal manifolds which is faithful and essentially surjective, but not full: i.e., which "forgets only structure", in the terminology of (Baez and Shulman, 2010, §2.4).

${ }^{25}$ (Weyl, 1918b, p. 396)

26(Weyl, 1918b, p. 397)

${ }^{27}$ Note that this term, now standard in the literature, is not used by Weyl himself, who speaks instead of a metrische Zusammenhang, literally: "metrical connection".
} 
with a length connection as a Weylian metric, and a manifold equipped with a Weylian metric as a Weylian manifold.

Returning to our main theme, the groundwork for Weyl's solution to the Problem of Space was laid already in his 1919 discussion:

Given the fundamental significance for the construction of geometry which, following recent investigations ..., attaches to the basic affine concept [affine Grundbegriff] of the infinitesimal parallel transport of a vector, the question in particular arises, whether the manifolds of the Pythagorean class of spaces [i.e., those in which the line element can be given in the Pythagorean form (1)] are the only ones which permit the establishment of this concept, and which correspondingly possess not only a metric, but also an affine connection. The answer is most likely affirmative, but a proof has so far not been rendered..$^{28}$

As already noted, it is this idea which drives Weyl's solution to the Problem of Space. More specifically, the key insight is that only the Pythagorean kind of spaces have the feature that they are associated with a unique concept of parallel transport. As is wellknown, for a given Riemannian metric there is a unique symmetric affine connection compatible with it; compatible, that is, in the sense that parallel-transported vectors retain their length. ${ }^{29}$ This result extends to Weylian manifolds: given a Weylian manifold, there is a unique compatible affine connection. ${ }^{30}$

This fact-the uniqueness of the affine connection, given the metrical structure-was greatly striking to Weyl, and something he put great emphasis on:

And now we come to that fact, already anointed above as the foundational fact of infinitesimal geometry, which brings the construction of geometry to a wonderfully harmonious conclusion. In a metrical space, there is one and only one way to formulate the concept of parallel transport so that ... this postulate is fulfilled: upon parallel transport of a vector, the interval determined by it should also remain unchanged. Thus, the principle of infinitesimal intervalor length-transfer, which underlies metrical geometry, automatically brings with it a principle of direction transfer; a metrical space naturally carries an affine connection. ${ }^{31}$

\footnotetext{
${ }^{28}$ (Riemann and Weyl, 1919, p. 27)

${ }^{29}$ (Malament, 2012, Lemma 1.9.1)

${ }^{30}$ (Folland, 1970, Theorem 2)

31(Weyl, 1923b, p. 124)
} 
At the heart of Weyl's solution to the Problem of Space is a demonstration that, among the much broader class of spaces obtained by allowing the line element to be an arbitrary homogeneous function of the differentials, only the Pythagorean spaces (i.e. the Weylian manifolds) will satisfy the following condition: 'whatever quantitative configuration (within the scope of the nature of the metric) the metric field may have assumed, it invariably and uniquely determines the affine connection. ${ }^{32}$

\section{Weyl's solution}

We turn now to a reconstruction of (part of) Weyl's argument. We follow the treatment given in (Weyl, 1923a): the text of a series of lectures on the Problem of Space which Weyl gave in the spring of 1922 in Barcelona and Madrid. There are some significant differences between the way in which Weyl carries out the argument here, compared to the way it is presented in his other work; ${ }^{33}$ moreover, this text has not been translated, and so we hope that the discussion here can help bring these ideas to a wider audience.

Weyl reaches his solution via a group-theoretic analysis. Given an $n$-dimensional Weylian manifold, of whatever nature, let us say that a linear automorphism $g$ of the tangent space at $P$ is congruent if it preserves the interval: that is, if for any vector $\xi$ at $P$, $f_{P}(g(\xi))=f_{P}(\xi)$. Since the composition of two congruent automorphisms will similarly be a congruent automorphism, as will the inverse of any congruent automorphism, the collection of all congruent automorphisms of $P^{\prime}$ s tangent space will form a group: let us refer to this group as the congruence group at $P$. For example, if $f_{P}$ is the Pythagorean function (1), then the congruence group will be the orthogonal group $O(n) .{ }^{34}$

Note that in general, the congruence group is not sufficient to determine the nature of the metric. For example, the 4-norm and the 6-norm on $\mathbb{R}^{2}$, i.e. the functions

$$
\|(x, y)\|_{4}:=\left(x^{4}+y^{4}\right)^{1 / 4}
$$

and

$$
\|(x, y)\|_{6}:=\left(x^{6}+y^{6}\right)^{1 / 6}
$$

respectively, have the same congruence group: the group consisting of right-angle

\footnotetext{
32(Weyl, 1923a, pp. 46-47)

${ }^{33}$ In particular, it does not involve the so-called "Postulate of Freedom": see Appendix A for discussion.

${ }^{34}$ Weyl uses the term 'Drehungsgruppe' for what we are calling the congruence group, which would more literally be translated as 'rotation group'; however (as Coleman and Korté (2001) note), the term 'rotation group' is nowadays almost exclusively used to refer to the groups $O(n)$ or $S O(n)$.
} 
rotations and reflections about the $x$ - and $y$-axes. ${ }^{35}$ Nevertheless, if the tangent space at $P$ has $O(n)$ as its congruence group, then the nature in question is Pythagorean (i.e. is given by a positive-definite quadratic form) at $P$. So if Weyl can find conditions which guarantee that each tangent space has $O(n)$ as its congruence group, then he will have succeeded in showing that only manifolds with a Pythagorean nature satisfy those conditions. ${ }^{36}$

In the seventh of his Barcelona lectures, Weyl argues that if we postulate uniqueness of parallel transport, then the congruence group's Lie algebra (i.e., the collection of infinitesimal congruent automorphisms $)^{37}$ must be of dimension $n(n-1) / 2$, and must satisfy a certain kind of antisymmetry condition (stated in more detail below). In the eighth and final lecture, Weyl sketches a proof that these conditions entail that the congruence group is the orthogonal group $O(n)$, and shows this by explicit calculation for the case $n=2$; a complete proof (for the case of arbitrary dimensions) is provided in the appendices. ${ }^{38}$

Weyl begins his argument by discussing the relationship between the metrical and the affine structure, i.e., between the transport of lengths and the transport of vectors. Recall that the length connection provides us with a unique way of transferring a lengthunit from a point $P$ to another point $P_{*}$ in $P^{\prime}$ s immediate neighbourhood, and hence of determining whether vectors $\xi$ at $P$ and $\xi_{*}$ at $P_{*}$ are the same length. In light of this, let us follow Weyl by saying that a linear isomorphism $\xi \in T_{P} M \rightarrow \xi_{*} \in T_{P_{*}} M$ is a congruent transport just in case it preserves the lengths of vectors: i.e., if the length of $\xi$ relative to a given length-unit at $P$ is the same as the length of $\xi_{*}$, relative to the transferral of that length-unit (using the length connection). Note that this is independent of the length-unit chosen at $P$.

At this stage we are restricting our attention to infinitesimal congruent transports: choosing some coordinate system around $P$, we are interested in those congruent trans-

\footnotetext{
${ }^{35}$ Coleman and Korté (2001) make the same observation.

${ }^{36}$ Coleman and Korté (2001) castigate much of the literature for failing to appreciate that Weyl's task concerned singling out the Pythagorean nature from the broader class of possible natures for the metric, not that of singling out $O(n)$ from the broader class of congruence groups (see, in particular, (Coleman and Korté, 2001, §§4.6-4.7)). However, it is not clear to us that this difference is as significant as they suggest, given that singling out the desired congruence group is a sufficient condition for singling out the desired nature of the metric.

${ }^{37}$ Weyl does not use the term "Lie algebra", but he notes that a collection of infinitesimal linear operations will form a linear family closed under the Lie bracket (again, not named as such): see (Weyl, 1923a, p. 50).

${ }^{38} \mathrm{~A}$ reconstruction of this second part of Weyl's solution, though certainly of value, is beyond the scope of this paper. The aspect of Weyl's proof is notoriously involved: Weyl describes himself as first having worked it out 'not through contemplation of the sense of the above-mentioned conditions, but rather only through mathematical acrobatics' (Weyl, 1922b, p. 120).
} 
ports $\xi \mapsto \xi_{*}$ for which

$$
\xi_{*}^{i}=\xi^{i}+d \xi^{i}
$$

Since congruent transports are linear, it follows that $d \xi^{i}=-\Lambda_{k}^{i} \xi^{k} \cdot{ }^{39}$ Let us say that the $\Lambda_{k}^{i}$ are the coefficients of the congruent transport. Now, without yet specifying the dimensionality of the manifold, suppose that $P_{*}$ is at the point $(\varepsilon, 0,0, \ldots, 0)$. If we let $\Lambda_{1 k}^{i}$ be the coefficients of the congruent transport from $P$ to $P_{*}$, and $\Lambda_{2 k}^{i}$ be the coefficients of a congruent transport from $P$ to the point $(0, \varepsilon, 0, \ldots, 0)$, then (for any $\alpha, \beta \in \mathbb{R})$ we can show that $\alpha \Lambda_{k 1}^{i}+\beta \Lambda_{k 2}^{i}$ are the coefficients of a congruent transport from $P$ to the point at $(\alpha \varepsilon, \beta \varepsilon, 0, \ldots, 0)$. Hence, once $n$ congruent transports $\Lambda_{k r}^{i}$ have been chosen, there is uniquely fixed a congruent transport to any point in $P^{\prime}$ s infinitesimal neighbourhood. As Weyl puts it:

... the formula,

$$
d \xi^{i}=-\Lambda_{k r}^{i} \xi^{k}(d x)^{r}
$$

supplies a system of infinitesimal congruent transports to the totality of points $P^{\prime}=\left(d x^{1}, d x^{2}, \ldots, d x^{n}\right)$ of the neighbourhood of $P .{ }^{40}$

Weyl's argument then runs as follows. Take a point $P_{0}$ in our manifold $M$, and introduce some coordinate system around it. Take as given the congruence group $G_{0}$ at $P_{0}$ (but not the congruence group at any other point). ${ }^{41}$ Now let $\Lambda_{k r}^{i}$ be an arbitrary collection of $n^{3}$ numbers. For every point $P$ in the infinitesimal neighbourhood of $P_{0}$, we can define a linear isomorphism from $T_{P_{0}} M$ to $T_{P} M$ by (6). Since every vector determines a length-unit (the one according to which that vector is of unit length), we can use this to define a length-unit transfer from $P_{0}$ to $P$, i.e. a length connection. Moreover, if we let the congruence group $G$ at $P$ be defined as the image of $G_{0}$ under this linear isomorphism, then the isomorphism will be congruence-preserving, and hence $\Lambda$ will represent a system of infinitesimal congruent transports.

Now that we have a length connection and congruence groups on the infinitesimal neighbourhood of $P_{0}$, any infinitesimal congruent transport from $P_{0}$ to $P$ may be obtained from a given such transport by composition with some infinitesimal congruent transformation. ${ }^{42}$ Consequently, any system of infinitesimal congruent transports may

\footnotetext{
${ }^{39}$ The minus sign is a matter of convention.

${ }^{40}$ (Weyl, 1923a, p. 48) Note that we have slightly altered Weyl's notation to fit with that of this essay.

${ }^{41}$ That is, we do not fix the action of the congruence group at other points; we know, from the fact that the nature of the metric is everywhere the same, that the congruence group at any other point will be isomorphic to $G_{0}$.

${ }^{42}$ I.e. an element of $G^{\prime}$ s Lie algebra.
} 
be obtained from our original system (that encoded by the $\Lambda_{j k}^{i}$ ) by specifying $n$ such infinitesimal congruent transformations: one for each of the $n$ linearly independent coordinate displacements $d x^{r}$. If we let $A_{k r}^{i}$ be the infinitesimal congruent transformation associated to $d x^{r}$, then the action of such a transformation on an arbitrary vector $\xi$ at $P$ is given (in terms of components) by:

$$
\xi^{i} \mapsto \xi^{i}-A_{k r}^{i} \xi^{k}(d x)^{r}
$$

It is at this point that we impose the postulate mentioned above, 'that among all these systems of infinitesimal congruent transports, a unique one is to be found which is simultaneously a possible system of parallel displacement'.43 It follows that there is a unique array of $A_{k r}^{i}$ which will bring about such a system of parallel transport. Using the Christoffel symbols now ubiquitous in general relativity, a parallel transport can be represented by $\Gamma_{j k}^{i}$, subject only to the symmetry requirement that $\Gamma_{j k}^{i}=\Gamma_{k j}^{i}$. We can then state Weyl's postulate as follows: given any $\Lambda_{j k}^{i}$, there is a unique system of parallel transport $\Gamma_{j k}^{i}$ and a unique system of infinitesimal congruent transformations $A_{j k}^{i}$ such that

$$
\Lambda_{j k}^{i}=\Gamma_{j k}^{i}-A_{j k}^{i}
$$

From this, Weyl proceeds to draw the following conclusions. First, if the dimensionality of the congruence group $G$ (and hence, of its Lie algebra $\mathfrak{g}$ ) is $N$, then since every $\Lambda_{j k}^{i}$ (with $n^{3}$ independent parameters) corresponds to a unique $\Gamma_{j k}^{i}\left(n^{2}(n+1) / 2\right.$ independent parameters) and $A_{j k}^{i}(n N$ independent parameters $)$, then $n^{3}=\frac{n^{2}(n+1)}{2}+n N$; that is,

$$
N=\frac{n(n-1)}{2}
$$

Second, note that if $A_{j k}^{i}=A_{k j}^{i}$, then $\Lambda_{j k}^{i}$ will represent a system of parallel transport, which must therefore be identical with that represented by $\Gamma_{j k}^{i}$-from which it follows that $A_{j k}^{i}=0$. So the family of the $A_{j k}^{i}$ has the feature that they are symmetric $\left(A_{j k}^{i}=A_{k j}^{i}\right)$ only if they all vanish. These two conclusions are the conditions on the congruence group's Lie algebra which-as discussed above-will lead us to the conclusion that the congruence group must be the orthogonal group (by an argument that we forebear from reconstructing here).

It bears emphasising how striking Weyl's achievement here is. Not only has he shown how the Problem of Space may be reframed in the light of general relativity; he has also shown that a satisfactory solution may be arrived at by the requirement that one's

${ }^{43}($ Weyl, 1923a, p. 49) 
standard of length-comparison uniquely fixes one's standard of direction-comparison. It should also be stressed that Weyl's analysis is independent of his unorthodox geometrical background, insofar as Riemannian geometry is (up to an arbitrary choice of global scale) a special case of Weylian geometry.

In the wake of general relativity, it is evident that something fundamental has shifted in the implicit assumptions built into our practices of describing space geometrically. Weyl's solution to the Problem of Space offers an insight into precisely this fundamental shift. For Weyl, the possibility of describing space in geometrical terms depends only on the possibility of an idealized observer at a point, "freely mobile" in the sense of being free to rotate at that point and start moving in any direction. Although such an observer can compare the dimensions of (infinitesimal) bodies in her immediate vicinity - that is determined only by the nature of space itself-what she might go on to discover about the larger-scale structure of space as she explores larger regions of it is left maximally unconstrained. ${ }^{44}$ Weyl sums up this new conception of space, implicit in general relativity as he understood it, with the following vivid metaphor:

Euclidean space may be compared to a crystal, built up of uniform unchangeable atoms in the regular and rigid unchangeable arrangement of a lattice; Riemannian space to a liquid, consisting of the same indiscernible unchangeable atoms, whose arrangement and orientation, however, are mobile and yielding to forces acting upon them..$^{45}$

\section{The status of the metric field}

Let us return to a consideration of what Weyl's work can contribute to the relatively recent debate over the status of the metric field. Recall that, on the one hand, because in general relativity the metrical field incorporates the gravitational field, some (including Earman, Norton and Rovelli) have argued that the metric tensor should be regarded as representing a physical field, akin to the electromagentic field. On such a view, it is the bare topological manifold, absent any metrical properties, that should be regarded as representing spacetime itself. On the other hand, others (including Maudlin and Hoefer) have argued that metrical structure remains at the heart of paradigmatic spatiotemporal structure.

\footnotetext{
${ }^{44}$ For the curious reader, it is this Weylian notion of an idealized observer, free to rotate and move in any direction, that inspired the title for this paper. (Thanks to Stephen Mackereth for the reminder that this title was, in fact, his idea.)

${ }^{45}$ (Weyl, 2009, p. 88)
} 
In considering how Weyl himself might have responded to this debate, the following statement seems unequivocal:

... it is not correct to say that space or the world is in itself, prior to any material content, merely a formless continuous manifold in the sense of analysis situs; the nature of the metric is peculiar to it in itself, only the mutual orientation of the metrics at various points is contingent, a posteriori and dependent on the material content. ${ }^{46}$

Thus, Weyl contrasts the nature of the metric - which, as we have seen, has its character fixed by the relationship between affine and metrical structure-with the orientation of the metric, encapsulating the remaining degrees of metrical freedom:

Thus one sees how the nature of the metric can be the same at every location, even while its quantitative determination, the-so to speak-mutual orientation of the metric at different points, is still very changeable and capable of continuously varying configurations. Thus, from this standpoint, the $a$ prioristic essence of space (defined by the nature of the metric) ... is divorced from the mutual orientation of the metric at the different points, which is a posteriori, i.e., contingent and naturally dependent on material content ... ${ }^{47}$

For present purposes, Weyl's distinction between the a priori and a posteriori serves to indicate the metrical properties that he attributes to space itself as contrasted with the metrical properties he attributes to the particular distribution of matter and energy. The fact that Weyl explicitly states that empty space is not 'merely a formless continuous manifold' would seem to place him squarely against the view advanced by Earman, Norton and Rovelli. But in fact Weyl's analysis allows for a distinction that none of the more contemporary protagonists have in view whilst capturing motivations from both sides. On the one hand, there is the awkwardness of regarding the dynamical aspects of the metric as attributable to space itself; on the other hand, there is the fact that a bare manifold seems genuinely insufficient to represent anything we would recognize as space. But Weyl's distinction between the nature and orientation of the metric field provides a way to retain the idea that space is intrinsically metrical without thereby being forced to attribute all the dynamical aspects of the metric field to space itself. ${ }^{48}$

${ }^{46}$ (Weyl, 1922b, p. 117)

${ }^{47}($ Weyl, 1922a, p. 216)

${ }^{48}$ One question we are left with here is whether the 'mutual orientation of the metric from point to point' should be regarded as in some sense akin to a physical field, and, if so, how. See Appendix B for a (partial) attempt at an answer. 
Weyl thus provides a "middle way" between bare-manifold and manifold-plus-metric accounts of spacetime, arguing that only the local metrical properties-properties which are independent of the variable distribution of matter and energy - are attributable to space itself.

One thing that emerges from the debate over the status of the metric field is that, in the wake of general relativity, we lack a principled means of identifying which mathematical structures represent features of space. By contrast, this was something that the figures of the nineteenth century had available to them: the classical solution to the Problem of Space provided a justification for why a particular collection of mathematical structures (the constant curvature geometries) could play this particular representational role. Once the classical solution became untenable, however, this justification went with it. Weyl's new solution to the Problem of Space thus offers a new justification for why an enlarged collection of mathematical structures - differential manifolds equipped with an infinitesimal Pythagorean-Riemannian metric-are candidate descriptions of physical space.

Throughout this paper, we have been treating Weyl's solution to the Problem of Space independently of his broader philosophical commitments. The fact that it is possible to do so points to the fundamental nature of the Problem itself. This is evident from the fact that, philosophical differences notwithstanding, there was broad agreement on the classical solution to the Problem of Space prior to general relativity. Poincaré, for example, could accept Helmholtz's solution to the Problem of Space whilst disagreeing with Helmholtz's claim that the value of the curvature of space would be determined by experiment. (For Poincaré, famously, the choice amongst constant curvature geometries was a matter of pure convention.) In a similar way, it is open to us to accept Weyl's new solution to the Problem of Space (and the insight into the conception of space implicit in general realtivity that Weyl offers) independently of Weyl's own broader philosophical commitments. ${ }^{49}$

Weyl's argument seeks to show that reflection upon the concept of (physical) metrical structure-in particular, upon the required relationship between metrical structure and affine structure-provides a justification for the Pythagorean nature of the metric. This provides a different argument for regarding the metric as encoding spatial structure, beyond merely noting that certain "paradigimatically spatial" properties depend upon it. In Weyl's analysis the sine qua non of physical geometry is that it realises a concordance between parallelism and congruence, and so the physical geometries are those

${ }^{49}$ For a detailed discussion of the philosophical commitments framing Weyl's approach to the Problem of Space, especially Weyl's interest in Husserlian phenomenology, see Ryckman (2005), §6. 
whose infinitesimal metrical structures uniquely determine affine structures over finite distances..$^{50}$ It is with this kind of insight in view that we urge that engaging with the Problem of Space remains important, not merely as providing a different answer to the question of what represents space, but rather as a means of shedding light on the question of what it is to represent space.

\section{Acknowledgments}

Our thanks to audiences at the 2015 Irvine-Pittsburgh-Princeton conference in Princeton, the 2015 meeting of the British Society for the Philosophy of Science in Manchester, and the 2019 Early Career Workshop in History and Philosophy of Physics in Pittsburgh. Thanks also to Gal Ben Porath, Stephen Mackereth, and an anonymous referee for their help in improving this paper. Special thanks to John Dougherty for contributing to this project across its various iterations and in particular for his detailed comments on an earlier draft.

\section{A. The 'Postulate of Freedom'}

In our reconstruction of Weyl's solution, we founded Weyl's argument on only one postulate, rather than two. In this, we diverge from the more standard view in the literature, namely that Weyl's proof is based upon both a Postulate of Coherence (essentially the uniqueness postulate used in our discussion), and a Postulate of Freedom: ${ }^{51}$ the postulate that any arbitrary collection $\Lambda_{k r}^{i}$ of $n^{3}$ numbers represents a possible system of congruent transport. Now, this claim features in our reconstructions of Weyl's proofbut as an observation about the geometry, not as an independently stated postulate. It is widely accepted that this is the correct logical status to give to the Postulate of Freedom: since the work of Scheibe (1957), it is standard to describe the (so-called) Postulate of Freedom as a theorem rather than a postulate. ${ }^{52}$ But what of its historical status? That is, did Weyl consider this to be a theorem or an independent postulate?

We submit that on this matter, Weyl changed his mind-but that the version given here represents his more mature view. Our reconstruction above follows the line of

\footnotetext{
${ }^{50}$ It is worth noting that this requirement also ensures that inertial structure can be identified unambiguously: this suggests a connection between Weyl's work and Knox's analysis of spacetime as whatever plays the functional role of determining inertial frames Knox (2017).

${ }^{51}$ See, for instance, Hawkins (2000), Scholz (2001), and Coleman and Korté (2001).

${ }^{52}$ See e.g. Scheibe (1988), (Scholz, 2001, p. 92). Coleman and Korté (2001) are an exception, seemingly because of how strongly they reject Scheibe's interpretation of the Problem of Space.
} 
thought given in Weyl (1923a), in which Weyl only ever refers to one postulate (not two), in which the "freedom of the metric" features as a preliminary observation, not a positive statement:

I come now to the synthetic part in the Kantian sense. It is necessary to precisely formulate the postulate suggested earlier, which shall determine the kind of rotation group which is characteristic for the actual world. First, the freedom guaranteed to it! The free deformability of the metrical field is available to such a degree that for a given rotation group at $P_{0}$, the metrical connection of this point with the points in its surroundings can always be so formed that the equation

$$
d \xi^{i}=-\Lambda_{k r}^{i} \xi^{k}(d x)^{r}
$$

with arbitrarily given coefficients $\Lambda_{k r}^{i}$, represents a system of infinitesimal congruent transport of the tangent space at $P_{0}$. Second, the positive part of the postulate: however this metrical connection of $P_{0}$ with the points in its surroundings may have been formed, among the possible systems of parallel displacements of the tangent space there is always a unique one which is simultaneously a system of infinitesimal congruent transport; the metrical connection uniquely determines the affine..$^{53}$

This is in sharp contrast to the version of this argument given elsewhere by Weyl, where-indeed - the postulates of freedom and coherence are distinguished from one another and separately stated. But that treatment represents an earlier phase of Weyl's thought: this discussion occurs first in the fourth edition of Raum, Zeit, Materie (Weyl, 1921), which he had completed by November 1920 (the date given in the preface, wherein he refers to 'a deeper group-theoretic formulation' of the Problem of Space as one of the major changes from the third edition); here, he succeeded in deriving the conditions on the Lie algebra, but not in showing that these conditions sufficed to uniquely identify the orthogonal group (except in the cases of two or three dimensions). A few months later, he had succeeded in completing the proof, for the case of arbitrary dimension: the proof was submitted in April 1921 (published as (Weyl, 1922b)), and Weyl discussed the result in a lecture of September 1921 (the text of which was published as (Weyl, 1922a)). ${ }^{54}$ All of these papers treat the Postulate of Freedom as a separate postulate, but all of them are prior to Weyl's delivery of the Spanish lectures (in spring 1922).

\footnotetext{
${ }^{53}$ (Weyl, 1923a, p. 49)

${ }^{54}$ This timeline is based upon (Hawkins, 2000, §11.2).
} 
That said, it is true that Weyl also distinguished the two postulates in the fifth edition of Raum, Zeit, Materie, which was completed in autumn 1922. However, he finished preparing the text of the lectures (involving their translation from French and Castilian back into German, and the inclusion of appendices completing the proof) in April 1923. ${ }^{55}$ Moreover, he appears to consider this text to supersede both (the treatment of the Problem of space in) the fifth edition, and the paper Weyl (1922b):

I think of this little monograph primarily as an expansion of the book "Raum, Zeit, Materie" (5th ed., Julius Springer 1923). The deeper understanding of the problem of space, drawing on group theory, was there only briefly touched upon ...; that is made good upon here....

For the inclusion of a complete proof of that main group-theoretic result, which the problem of space leads us onto, I decided in the first instance ... to simplify the first proof (Mathematische Zeitschrift 12, p. 114) to a great extent.

For these reasons, it seems to us appropriate to take the one-postulate version of the argument as more reflective of Weyl's considered opinion.

Incidentally, there is an interesting question of the extent to which the published 1923 text reflects the lectures as they were delivered: in his preface, Weyl refers to the text as 'containing [the lectures] almost verbatim', but also remarks that the eighth lecture 'has had to undergo a sweeping revision.' Light could perhaps be shed on this matter if the projected Catalan version of the lectures had been published by the Institut d'Estudis Catalans, as was apparently planned (and happened for similar invited lecturers, e.g. Levi-Civita (1922)); however, it appears that this never came to fruition. ${ }^{56}$ The document list for the Weyl archive at the ETH Zurich ${ }^{57}$ does not appear to list notes for these lectures; it does, however, mention correspondence between Weyl and the Institut d'Estudis Catalans, and between Weyl and Esteban Terradas (who had invited Weyl to Barcelona, and who edited the series in which the book would have appeared), which could perhaps offer an account of why the Catalan book was never published.

\footnotetext{
55(Weyl, 1923a, p. III)

${ }^{56}$ There is no reference to such a volume in the Weyl bibliography of Newman (1957), nor in the catalog of the Institut d'Estudis Catalan (Institut d'Estudis Catalans, 1997).

${ }^{57}$ Handschriften und Autographen der ETH-Bibliothek (1995)
} 


\section{B. The nature of the metric as background object}

As discussed in $\S 3$, the kind of homogeneous function used to express the norm at each tangent space specifies the nature of the metric: that is, if the interval is expressed in some coordinate system by the homogeneous function $f$, then the metric as nature $(f)$ (where $(f)$ is the equivalence class of $f$ under arbitrary linear transformations). How do we capture the nature of a metric in more coordinate-free terms?

To this end, let us start by considering what it would be for two conformal manifolds to be of the same nature. If two conformal manifolds both have nature $(f)$, then for any points $p \in M$ and $p^{\prime} \in M^{\prime}$, there exist coordinate systems $U \rightarrow \mathbb{R}^{n}$ and $U^{\prime} \rightarrow \mathbb{R}^{n}$ (where $p \in U$ and $p^{\prime} \in U^{\prime}$ ) such that the intervals at $p$ and $p^{\prime}$ are both expressed by $f$. But it then follows that there is a linear bijection $h: T_{p} M \rightarrow T_{p^{\prime}} M^{\prime}$ which preserves congruence. Conversely, if there exists such a map $h$, then given a coordinate system around $p$ in which $f$ expresses the interval at $p$, we can push forward the coordinate basis on $T_{p} M$ to $T_{p^{\prime}} M^{\prime}$ under $h$, and then find a coordinate system around $p^{\prime}$ for which the pushed-forward basis is the coordinate basis; by construction, this basis will be one relative to which the interval at $p^{\prime}$ is expressed by $f$. Thus, two metrics are of the same nature if and only if there is a congruence-preserving map from a tangent space in one to a tangent space of the other.

Following this line of thought, we can represent the nature of the metric as follows. Suppose that we begin with an $n$-dimensional manifold $M$. Consider a fibre bundle which is isomorphic, qua vector bundle, to the tangent bundle $T M$; this bundle is distinguished from the tangent bundle by the fact that there is no privileged identification of points in the bundle with directional derivatives on the manifold. Now let $W$ be the result of equipping this fibre bundle with a faithful action of $O(n)$ on each fibre (so $O(n)$ is the structure group of $W$ ). Let us (tendentiously) say that $W$ is a natured manifold. Any conformal manifold can be regarded as a natured manifold, by letting the action of $O(n)$ on any tangent space be the group of congruence-preserving maps; and by the argument above, two conformal manifolds will be isomorphic qua natured manifolds just in case they are of the same nature. So the name is appropriate, and we can think of $W$ as a manifold equipped with a nature.

To turn $W$ into a conformal manifold, we need to specify (in a smoothly varying fashion) a pointwise linear bijection $e: W \rightarrow T M$. Such a field $e$ is known as a solder form or vielbein: in the context of general relativity, it is often called a tetrad field. ${ }^{58}$ As before, to move from a conformal manifold to a Weyl manifold, we require also a

\footnotetext{
${ }^{58}$ See Weatherall (2016) for discussion of solder forms in general relativity.
} 
length connection. Thus, on this reading, the nature of the metric is expressed in the congruence-structure of an auxiliary internal bundle; the orientation is captured by a tetrad field and a length connection.

We are not the only ones to have suggested that unpacking Weyl's distinction between nature and orientation might be associated with the vielbein formalism: for example, Ryckman makes the following suggestive remarks:

The coordinate systems of the local group are defined in the tangent space over each point: each one is an orthonormal frame called a tetrad which is free to rotate independently of the tetrads over the other points. ... The effects of gravity are "restored" by a connection (here, the gravitational potential) which reconciles the local laws on various points. This is the distinction pointed to by Weyl in a purely mathematical context between the nature and the orientation of the metric ... ${ }^{59}$

This suggests, in contrast to our reading, that the nature of the metric be identified with the tetrad field (together with the internal metrical structure), rather than just the internal metrical structure: this would amount to identifying the nature of the metric with the conformal structure, and the orientation with the (length) connection.

In defence of our reading, we note not only that it is the most natural fit with Weyl's definition of nature in terms of equivalence classes of homogeneous functions, but also that it fits best with Weyl's insistence on the fixed and a priori character of the nature. We touched upon this already, but it is helpful to consider how Weyl introduces the distinction between nature and orientation in the 5th edition of Raum-Zeit-Materie:

The nature of the metric signifies the aprioristic essence of space in its metrical aspect; it is one, thus it is also absolutely determined and does not partake of the irrevocable vagueness of those which occupy a variable place in a continuous scale. What is not determined through the essence of space, but rather is a posteriori (i.e. contingent, intrinsically free, and capable of arbitrary virtual changes), is the mutual orientation of the metrics at different points ... ${ }^{60}$

With regards to this distinction, it is surely more natural to place conformal structure on the a posteriori side, given that Weylian manifolds (including Riemannian manifolds)

\footnotetext{
${ }^{59}$ (Ryckman, 1999, p. 596). It may be noted that Ryckman identifies a tetrad as a frame field, i.e., a choice of basis at each point, rather than as a pointwise linear isomorphism between an internal bundle and the tangent bundle. The two definitions are equivalent if a preferred frame field for the internal bundle is chosen.

${ }^{60}$ (Weyl, 1923b, pp. 102-103)
} 
can have very different conformal structures-as Weyl was surely aware, having introduced the conformally invariant Weyl curvature in Weyl (1918b). By contrast, the nature as we have characterised it is a local invariant in the following sense: given any two Weylian manifolds, expressed in vielbein form as $(\pi: W \rightarrow M, e)$ and $\left(\pi^{\prime}: W^{\prime} \rightarrow M^{\prime}, e^{\prime}\right)$, for any $p \in M$ and $p^{\prime} \in M^{\prime}$, there are neighbourhoods $U \ni p$ and $U^{\prime} \ni p^{\prime}$ such that there is an $O(n)$-bundle automorphism $\phi:\left.\left.W\right|_{U} \rightarrow W^{\prime}\right|_{U^{\prime}}$.

Phrased in these terms, the distinction that Weyl articulates as the $a$ priori versus $a$ posteriori properties of space starts to sound like a rather more familiar distinction in the contemporary literature on spacetime theories: the Anderson-Friedman distinction between absolute and dynamical structures. ${ }^{61}$ To formulate this distinction, Friedman first introduces the concept of $d$-equivalence: given a pair of models $\left\langle M, \Phi_{1}, \ldots, \Phi_{n}\right\rangle$ and $\left\langle M, \Psi_{1}, \ldots, \Psi_{n}\right\rangle$ of some space-time theory $T, \Phi_{i}$ and $\Psi_{i}$ are $d$-equivalent just in case 'for every $p \in M$, there are neighbourhoods $A, B$ of $p$, and a transformation $h: A \rightarrow B$, such that $\Psi_{i}=h \Phi_{i}$ on $A \cap B .^{\prime 62}$ Then,

A geometrical object $\Phi_{i}$ is an absolute object of a space-time theory $T$ just in case for any two models $\left\langle M, \Phi_{1}, \ldots, \Phi_{n}\right\rangle$ and $\left\langle M, \Psi_{1}, \ldots, \Psi_{n}\right\rangle$ of $T, \Phi_{i}$ and $\Psi_{i}$ are $d$-equivalent. ${ }^{63}$

As is well-known, general relativity admits no absolute objects in this sense. But there is a natural way of modifying Friedman's definition of $d$-equivalence to apply to theories with internal bundles. Given a pair of bundles with fields $\left(\pi: E \rightarrow M, \Phi_{1}, \ldots, \Phi_{n}\right)$ and $\left(\pi: E \rightarrow M, \Psi_{1}, \ldots, \Psi_{n}\right)$, let us say that $\Phi_{i}$ and $\Psi_{i}$ are b-equivalent if for every $p \in M$, there are neighbourhoods $A, B$ of $p$, and a bundle automorphism $h:\left.\left.E\right|_{A} \rightarrow E\right|_{B}$, such that $\Psi_{i}=h \Phi_{i}$ on $\left.\left.E\right|_{A} \cap E\right|_{B}$; we can then define an absolute object as one which is $b$-equivalent across any pair of models, rather than $d$-equivalent.

Hence, if we write any Weylian geometry as $(\pi: E \rightarrow M, N, e)$ (where $N$ is the nature, i.e. the action of $O(n)$ on each fibre) then it follows from the observation above that for any models $\left(\pi: E \rightarrow M, N^{\prime}, e^{\prime}\right), N$ and $N^{\prime}$ are $b$-equivalent; and hence, that the nature of the metric is an absolute object in (a natural analogue of) Friedman's sense. So this analysis shows that, even in light of the great conceptual changes wrought by general relativity upon our concepts of space, something fixed and absolute remains. As Weyl puts it:

${ }^{61}$ Although this terminology is standard, it is not clear to what extent Anderson's concept of absolute object (as described in (Anderson, 1967, §4.3)) coincides or overlaps with Friedman's (introduced below): for discussion, see Pitts (2006).

62(Friedman, 1983, p. 58)

63(Friedman, 1983, p. 60) 
One sees that the Riemannian viewpoint does not abnegate the existence of an aprioristic element in spatial structure; only the boundary between the a priori and the a posteriori is shifted. ${ }^{64}$

With this in view, let us return once more to the debate over what represents spacetime. We have seen that the nature of the metric may be understood as a form of structure which goes beyond that of the bare manifold, but is nevertheless absolute or a priori; it in this sense that it represents an intermediate level of structure between the metric and manifold. That said, it will not succeed in doing the kinds of things that Maudlin or Hoefer argued above that space(time) structure should do. If one is given a manifold with a nature, then there is no way to compute the length of a curve from one point to another; one needs a vielbein field in order to be able to do that. So those who take the metric to be spatiotemporal in character will be unlikely to be persuaded that the (mere) nature of the metric represents spacetime.

\section{References}

Anderson, J. L. (1967). Principles of Relativity Physics. Academic Press, New York.

Baez, J. C. and Shulman, M. (2010). Lectures on n-categories and cohomology. In Baez, J. C. and May, J. P., editors, Towards Higher Categories, pages 1-68. Springer.

Coleman, R. A. and Korté, H. (2001). Hermann Weyl: Mathematician, Physicist, Philosopher. In Scholz, E., editor, Hermann Weyl's Raum-Zeit-Materie and a General Introduction to His Scientific Work, pages 198-270. Springer.

Curiel, E. (2017). On Geometric Objects, the Non-Existence of a Gravitational StressEnergy Tensor, and the Uniqueness of the Einstein Field Equation. Studies in History and Philosophy of Science Part B: Studies in History and Philosophy of Modern Physics. Forthcoming. References are to draft of February 2nd, 2017.

Dewar, N. and Weatherall, J. O. (2018). On Gravitational Energy in Newtonian Theories. Foundations of Physics, 48(5):558-578.

Earman, J. and Norton, J. (1987). What Price Spacetime Substantivalism? The Hole Story. The British Journal for the Philosophy of Science, 38(4):515-525.

Einstein, A. (1921). Geometrie und Erfahrung. Springer Berlin Heidelberg, Berlin, Heidelberg.

${ }^{64}$ (Weyl, 1923b, p. 103) 
Folland, G. B. (1970). Weyl manifolds. Journal of Differential Geometry, 4(2):145-153.

Friedman, M. (1983). Foundations of Space-Time Theories: Relativistic Physics and Philosophy of Science. Princeton University Press, Princeton, NJ.

Handschriften und Autographen der ETH-Bibliothek (1995). Hermann Weyl: Verzeichnis der Manuskripte und Korrespondenzen.

Hawkins, T. (2000). Emergence of the Theory of Lie Groups. Springer New York, New York, NY.

Helmholtz, H. v. (1971). On the Origin and Significance of Geometric Axioms (1870). In Selected Writings of Hermann von Helmholtz. Wesleyan University Press, Middletown, CT.

Helmholtz, H. v. (2007). On the factual foundations of geometry (1866). In Pesic, P., editor, Beyond geometry: Classic papers from Riemann to Einstein, pages 47-52. Dover.

Hoefer, C. (1996). The metaphysics of space-time substantivalism. The Journal of Philosophy, 93(1):5-27.

Institut d'Estudis Catalans (1997). Catàleg de publicacions, 1907-1996. Institut d'Estudis Catalans.

Knox, E. (2017). Physical relativity from a functionalist perspective. Studies in History and Philosophy of Science Part B: Studies in History and Philosophy of Modern Physics.

Levi-Civita, T. (1917). Nozione di parallelismo in una variatà qualunque. Rend. del Circ. Math. di Palermo, 42.

Levi-Civita, T. (1922). Qüestions de Mecànica Clàssica i Relativista: Conferències Donades El Gener de 1921. Col-lecció de Cursos de Física i Matemàtica. Institut d'Estudis Catalans, Barcelona.

Malament, D. B. (2012). Topics in the Foundations of General Relativity and Newtonian Gravitation Theory. University of Chicago Press, Chicago, IL.

Maudlin, T. (1988). The Essence of Space-Time. PSA: Proceedings of the Biennial Meeting of the Philosophy of Science Association, 1988:82-91.

Newman, M. H. A. (1957). Hermann Weyl. 1885-1955. Biographical Memoirs of Fellows of the Royal Society, 3:305-328. 
Pitts, J. B. (2006). Absolute objects and counterexamples: Jones-Geroch dust, Torretti constant curvature, tetrad-spinor, and scalar density. Studies in History and Philosophy of Science Part B: Studies in History and Philosophy of Modern Physics, 37(2):347-371.

Poincaré, H. (1898). On the Foundations of Geometry. Monist, 9(1).

Poincaré, H. (1952). Science and Hypothesis. Dover.

Read, J. (2018). Functional Gravitational Energy. The British Journal for the Philosophy of Science. Forthcoming.

Riemann, B. and Jost, J. (2013). Über Die Hypothesen, Welche Der Geometrie Zu Grunde Liegen: Historisch Und Mathematisch Kommentiert von Jürgen Jost. Springer, Berlin.

Riemann, B. and Weyl, H. (1919). Über Die Hypothesen, Welche Der Geometrie Zu Grunde Liegen. Springer, Berlin.

Rovelli, C. (1997). Half way through the woods. In Earman, J. and Norton, J. D., editors, The Cosmos of Science, pages 180-223. University of Pittsburgh Press.

Ryckman, T. (2005). The Reign of Relativity: Philosophy in Physics 1915-1925. Oxford University Press, USA.

Ryckman, T. A. (1999). Einstein, Cassirer, and General Covariance - Then and Now. Science in Context, 12(4):585-619.

Scheibe, E. (1957). Über das Weylsche Raumproblem. Journal für die reine und angewandte Mathematik, 197:162-207.

Scheibe, E. (1988). Hermann Weyl and the Nature of Spacetime. In Deppert, W., Hübner, K., Oberschelp, A., and Weidenmann, V., editors, Exact Sciences and Their Philosophical Foundations, pages 61-82. Lang, Frankfurt am Main.

Scholz, E. (2001). Weyls Infinitesimalgeometrie, 1917 - 1925. In Scholz, E., editor, Hermann Weyl's Raum-Zeit-Materie and a General Introduction to His Scientific Work, volume 30, pages 48-104. Birkhäuser Basel, Basel.

Scholz, E. (2016). The problem of space in the light of relativity: The views of Hermann Weyl and Elie Cartan. In Bioesmat-Martagon, L., editor, Eléments d'une Biographie de l'espace Géométrique, pages 255-312. Presses Universitaires de Nancy, Nancy.

Weatherall, J. O. (2016). Fiber bundles, Yang-Mills theory, and general relativity. Synthese, 193(8):2389-2425. 
Weyl, H. (1918a). Gravitation und Elektrizität. Preussische Akademie der Wissenschaften (Berlin) Sitzungsberichte. Physikalisch-Mathematike Klasse, pages 465-480.

Weyl, H. (1918b). Reine Infinitesimalgeometrie. Mathematische Zeitschrift, 2(3-4):384-411.

Weyl, H. (1921). Raum, Zeit, Materie: Vorlesungen über allgemeine Relativitätstheorie. Springer Berlin Heidelberg, Berlin, Heidelberg, 4th edition.

Weyl, H. (1922a). Das Raumproblem. Jahresbericht der Deutschen MathematikerVereinigung, 31:205-221.

Weyl, H. (1922b). Die Einzigartigkeit der Pythagoreischen Maßbestimmung. Mathematische Zeitschrift, 12(1):114-146.

Weyl, H. (1923a). Mathematische Analyse des Raumproblems. Springer, Berlin.

Weyl, H. (1923b). Raum, Zeit, Materie: Vorlesungen über allgemeine Relativitätstheorie. Springer, Berlin, 5 th edition.

Weyl, H. (2009). Philosophy of mathematics and natural science. Princeton University Press. 